\title{
Long-term effects of serial anodal tDCS on motion perception in subjects with occipital stroke measured in the unaffected visual hemifield
}

\author{
M. C. Olma ${ }^{1}$, R. A. Dargie ${ }^{2}$, J. R. Behrens ${ }^{1}$, A. Kraft ${ }^{1}$, K. Irlbacher ${ }^{1}$, M. Fahle ${ }^{3}$ and S. A. Brandt ${ }^{1}$ \\ ' Department of Neurology, Charité University Hospital, Berlin, Germany \\ ${ }^{2}$ The College of Medicine and Veterinary Medicine, The University of Edinburgh, Edinburgh, UK \\ ${ }^{3}$ Department of Human Neurobiology, University of Bremen, Bremen, Germany
}

\section{Edited by: \\ Janine Reis, \\ Albert-Ludwigs-University Freiburg, \\ Germany}

\section{Reviewed by:}

Carlo Miniussi, University of Brescia, Italy

Donatella Spinelli, Università di Roma Foro Italico, Italy

Andrea Antal, University Medical

Center Goettingen, Germany

*Correspondence:

M. C. Olma, Clinic for Neurology, Charité Universitätsmedizin Berlin, Charitéplatz 1, 10117 Berlin, Germany e-mail:manuel.olma@charite.de

\begin{abstract}
Transcranial direct current stimulation (tDCS) is a novel neuromodulatory tool that has seen early transition to clinical trials, although the high variability of these findings necessitates further studies in clinically relevant populations. The majority of evidence into effects of repeated tDCS is based on research in the human motor system, but it is unclear whether the long-term effects of serial tDCS are motor-specific or transferable to other brain areas. This study aimed to examine whether serial anodal tDCS over the visual cortex can exogenously induce long-term neuroplastic changes in the visual cortex. However, when the visual cortex is affected by a cortical lesion, up-regulated endogenous neuroplastic adaptation processes may alter the susceptibility to tDCS. To this end, motion perception was investigated in the unaffected hemifield of subjects with unilateral visual cortex lesions. Twelve subjects with occipital ischemic lesions participated in a within-subject, shamcontrolled, double-blind study. MRI-registered sham or anodal tDCS (1.5 mA, $20 \mathrm{~min})$ was applied on five consecutive days over the visual cortex. Motion perception was tested before and after stimulation sessions and at 14- and 28-day follow-up. After a 16-day interval an identical study block with the other stimulation condition (anodal or sham tDCS) followed. Serial anodal tDCS over the visual cortex resulted in an improvement in motion perception, a function attributed to MTN5. This effect was still measurable at 14-and 28-day follow-up measurements. Thus, this may represent evidence for long-term tDCS-induced plasticity and has implications for the design of studies examining the time course of tDCS effects in both the visual and motor systems.
\end{abstract}

Keywords: transcranial direct current stimulation, visual system, motor system, translational research, motion perception, neuroplasticity, learning

\section{INTRODUCTION}

Arguably the greatest future challenge facing transcranial direct current stimulation (tDCS), a promising tool for non-invasive neuromodulation, will be its effective translation to clinical use. However, evidence suggests that changes in connectivity and neuroplasticity in the aging or lesioned brain may have different prerequisites and mechanisms than similar processes in younger healthy systems (DeCarli et al., 2012; Grady, 2012). This raises the question of how directly tDCS-induced synaptic plasticity documented by numerous studies in animals and healthy young subjects will manifest in these subject groups, and to what extent persistent, long-term (weeks-months) and clinically relevant effects can be achieved.

The effects of tDCS on the human motor-cortex are wellestablished. Single-session stimulation with weak anodal or cathodal transcranial direct current (current densities of $<0.1 \mathrm{~mA} / \mathrm{cm}^{2}$ ) respectively lowers and raises the threshold to motor-evoked potential (MEP) induction by transcranial magnetic stimulation (TMS), with effects outlasting the stimulation period by up to $90 \mathrm{~min}$ (Priori et al., 1998; Nitsche and Paulus, 2000, 2001;
Rosenkranz et al., 2000; Quartarone et al., 2004; Furubayashi et al., 2008).

Pharmacological studies deepened our understanding of these effects and their relation to other forms of neuroplasticity (Gartside, 1968; Bailey et al., 2000; Liebetanz et al., 2002; Nitsche et al., 2003a, 2004). These collective findings strongly suggest that LTPlike and LTD-like processes underlie the observed neuroplastic effects of tDCS (Nitsche et al., 2008; Stagg and Nitsche, 2011).

Early animal work into direct current on exposed cortical surfaces reported similar findings in the motor and visual systems (Bindman et al., 1962, 1964; Creutzfeldt et al., 1962; Landau et al., 1964). TDCS studies in humans could demonstrate that excitability of the visual cortex, measured, e.g., by visual-evoked potentials (VEPs), can be elevated by occipital anodal tDCS and lowered by cathodal tDCS especially when low signal-to-noise stimuli are used (Antal et al., 2004a; Accornero et al., 2007; Lang et al., 2007). Studies utilizing low signal-to-noise perceptual threshold tasks detected significant anodal tDCS effects on visual contrast sensitivity (Kraft et al., 2010a; Olma et al., 2011), while a similar study identified no stimulation effects of anodal tDCS when supra-threshold 
stimuli were used (Antal et al., 2001). This suggests that threshold measures are well-suited to further assess tDCS in this system. Although the immediate effects of tDCS in the healthy visual system are of shorter duration than in the motor system - likely to be related to the interaction of current fields with gross cranial anatomy, cortical microarchitecture, and underlying system activity (Nitsche et al., 2008) - long-term effects of repeated tDCS may be translatable from the motor to the visual system (Antal and Paulus, 2008; Antal et al., 2011).

To this end, we investigated the immediate and long-term effects of anodal serial tDCS on an established motion detection threshold paradigm (Antal et al., 2004c; Kraft et al., 2010b). However, endogenous neuroplastic adaptation processes following a cortical lesion in the visual cortex may be up-regulated and alter the susceptibility to tDCS. Therefore, we investigated subjects suffering chronic visual-system ischemic strokes to model the presence of lesions in target stimulation populations. MRI-navigated anodal or sham tDCS was administered on five consecutive days over the ipsilesional visual cortex. Motion perception thresholds of the unaffected visual field (corresponding the contralesional hemisphere, to provide data comparable with previous studies in healthy subjects) were measured directly before and after each stimulation session, then again at 2- and 4-week follow-up after the fifth stimulation day, within a double-blinded, within-subject cross-over study.

\section{MATERIALS AND METHODS STUDY PARTICIPANTS}

Twelve subjects [mean age $53.5 \pm 14.9$ (SD) years; six female; all right-handed] participated in the study. All had a history of ischemic stroke and chronic homonymous visual field defects (hVFD; four homonymous hemianopias, seven homonymous quadrantanopias, one homonymous paracentral scotoma). In all subjects, no transient or persistent ischemic attacks had been reported or diagnosed in the 6 months preceding inclusion in the study. Subjects were thus in comparable chronic post-stroke phases, when nil or minimal spontaneous changes in visual perception are expected (Zhang et al., 2006). Mean interval between stroke occurrence and inclusion in the study was $18.4 \pm 8.2$ (SD) months. Occipital lesions were relatively small, well-defined unilateral posterior infarcts and - importantly - there remained intact cortex at the occipital pole (stimulation site): mean lesion volume was $8.4( \pm 7.0 \mathrm{SD}) \mathrm{cm}^{3}$ and mean minimum distance from the internal surface of the occipital pole cranium was $14.6( \pm 6.9 \mathrm{SD})$ $\mathrm{mm}$. Subjects had no history of hemorrhagic or traumatic brain injury, or progressive neurological disease, no ophthalmological or pre-chiasmal disorders, no psychiatric disease, cognitive impairment [as measured by Montreal Cognitive Assessment (MoCA, Nasreddine et al., 2005)], or history of substance abuse. Unilateral hemispatial neglect was excluded by assessment with clockdrawing, image-replication, line-bisection, and star-cancelation tasks. Each subject scored $\geq 3$ out of 5 in the visuo-spatial section and $\geq 4$ out of 6 in the attention section of the MoCA. Full function of the dominant (right) hand was present in all subjects. No subjects had metal cranial implants, cardiac pacemakers, or other implanted devices. Prior to inclusion in the study, standard automated perimetry confirmed congruent, homonymous visual field defects in all subjects. Standard 10-20 electroencephalogram of each subject was free of epileptiform potentials at rest and on graded photic and hyperventilation provocation. All subjects had normal or corrected-to-normal visual acuity and had not received structured visual rehabilitation. The study conformed to the Declaration of Helsinki and was approved by the local ethics committee. Each subject provided written, informed consent and received financial compensation to cover travel costs to appointments.

\section{EXPERIMENTAL PROCEDURE}

Experiments were performed before 12 noon to control for daily fluctuations in concentration and visual discrimination (Bonnefond et al., 2003; Schmidt et al., 2007). All experiments were performed in the same darkened room. At the start of each experimental test day, subjects sat quietly for 5 min to allow dark adaptation. The order of experimental procedure was as follows: computerized campimetric tests [i.e., performed on a flat-screen, not a perimetric bowl (Kraft et al., 2010b)] of color and motion detection ( $3 \mathrm{~min}$ each), automated threshold perimetry ( $6 \mathrm{~min}$ ), $20 \mathrm{~min}$ intervention (anodal or sham tDCS), perimetric tests (6 min), and computerized campimetric tests of color and motion perception ( 3 min each). The order of color and motion campimetric tests was randomized daily and balanced over the subject group. Results from the color campimetric test and contrast perimetry shall be reported elsewhere.

\section{STIMULI, TASK, AND PROCEDURE}

Details of the alternative forced-choice motion detection paradigm used have been previously published (Kraft et al., 2010b) and are thus only briefly outlined here. The test was performed on a $21^{\prime \prime}$ diameter, $1600 \times 1200$ pixel, $75 \mathrm{~Hz}$ Iiyama monitor connected to a personal computer. Subjects' heads were stabilized on an adjustable chin rest $60 \mathrm{~cm}$ from the monitor, the height of which was adjusted so that subject eye level was at the height of the monitor's central point. Subjects were required to maintain fixation of a small (12.0 arcmin) central dot throughout the full duration of both campimetric tests.

The stimuli for the motion detection task comprised a gray background $\left(54 \mathrm{~cd} / \mathrm{m}^{2}\right)$, against which 20,000 black dots of $5.0 \mathrm{arcmin}$ diameter moved to the right at a velocity of $3 \%$. A monochromatic circle of 210.0 arcmin diameter, composed of dots of the same two intensities but moving to the left with variable velocity, was presented for $200 \mathrm{~ms}$ in one (random) visual field quadrant at $5^{\circ}$ eccentricity from fixation (Figure 1).

Subject's task was to indicate the location of the circle via a manual button press using a four-button keypad (four-alternativeforced-choice task). After stimulus presentation, subjects had unlimited time to select the quadrant they deemed the stimulus was presented in. Incorrect responses triggered a short tone as error feedback, and each response elicited the next stimulus at an interval of $500 \mathrm{~ms}$. Stimulus dots were initially set to move to the left with a velocity of $3 \%$, producing an easily detectable circular stimulus (Figure 1). As the test progressed and subjects provided correct answers in each quadrant, the difference between the motion direction of background dots and stimulus dots (i.e., stimulus/background contrast) decreased, and stimuli became more difficult to detect. 


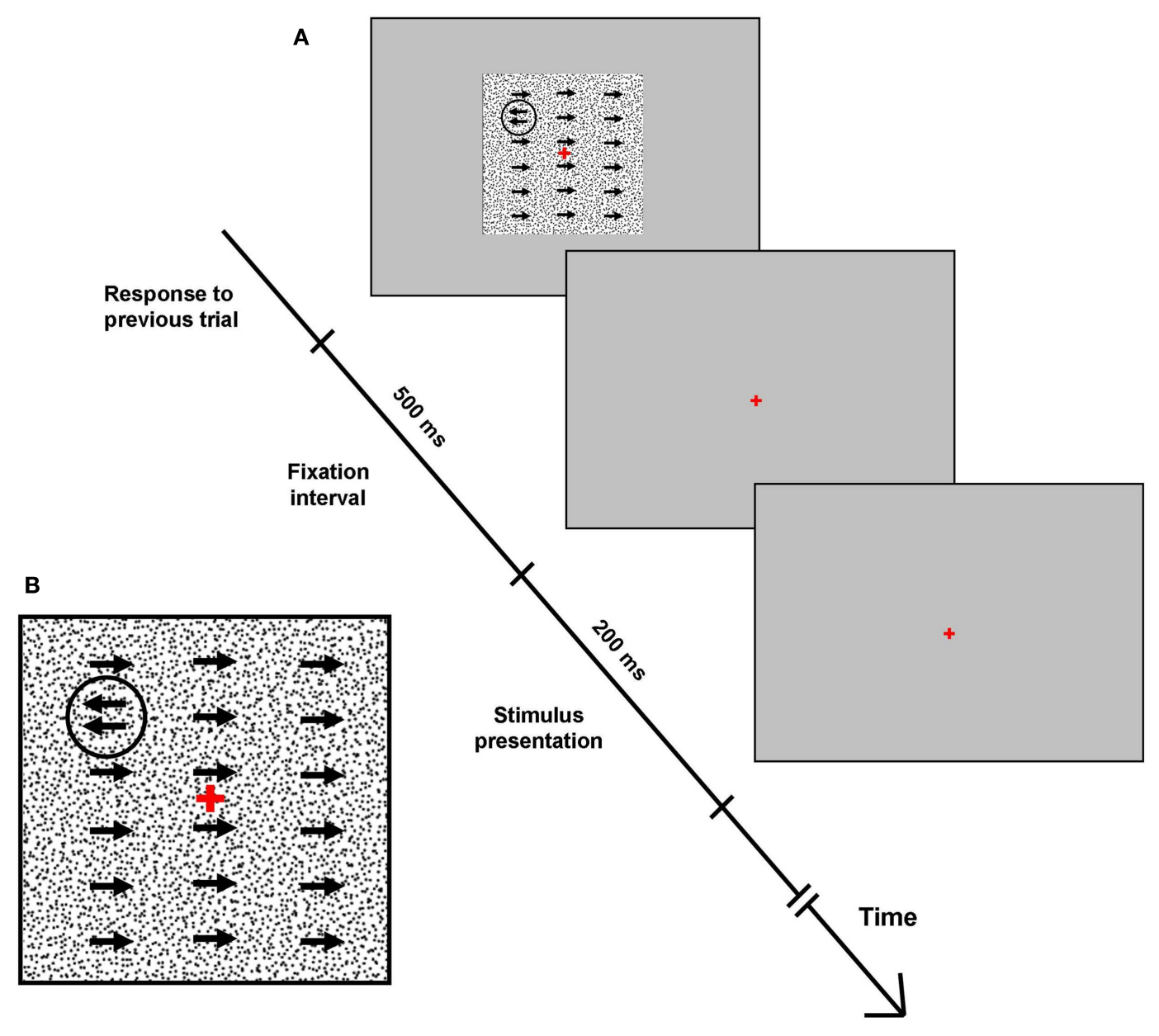

FIGURE 1 | Motion detection task. (A) Full-screen screenshots of the campimetric motion detection perceptual task. Each response was followed by a $500 \mathrm{~ms}$ fixation screen, before the test screen with background and quadrant-specific stimulus were presented for $200 \mathrm{~ms}$ (see text for stimulus and background details). Following this, subjects had unlimited time to select one quadrant via a four-button keypad. Arrows signify coherent movement of 5 acrmin diameter monochromatic dots. (B) Inset: close-up of test screen.
This allowed a quadrant-specific motion detection threshold to be attained, the contrast level (in degrees of stimulus movement direction) at which $62.5 \%$ responses were correct, based on the adaptive staircase procedure Quick Estimation by Sequential Testing (QUEST) (Watson and Pelli, 1983; Kraft et al., 2010b). All tests comprised 120 trials (30 per visual field quadrant) and lasted approximately $3 \mathrm{~min}$. The test was performed binocularly. No formal fixation control was applied, but the short stimulus presentation duration $(200 \mathrm{~ms})$ and investigator eye-movement observation via an adjustable mirror provided basic controls. Subjects were reassured that it was normal and necessary not to perceive all stimuli and encouraged to give intuitive responses, using all quadrants, when unsure. This aimed to reduce bias toward any particular quadrant. Nevertheless, the probability of guessing the right target quadrant was theoretically $25 \%$, with a measured standard deviation of $6.8 \%$ over the entire group.

However, some subjects with stroke-related visual field defects in specific quadrants could not or could only poorly perceive the motion stimuli there. Grouping "perceivers," "poor-perceivers," and "not-perceivers" into one group would cause a high heterogeneity confounding the interpretation of the tDCS effect in these ipsilesional quadrants. Therefore, data from the ipsilesional quadrants were excluded from the analysis in this study.

Threshold values for quadrants that subjects selected too seldomly for a threshold to be accurately calculated, or in which a threshold value greater than the highest possible value $\left(180^{\circ}\right)$ was calculated, were replaced with the value corresponding to the first stimulus presented, $180^{\circ}$ (Kraft et al., 2010b). Perceptual thresholds were conducted using post hoc fitting procedures (Probit-analysis). Age-corrected threshold values were obtained for all datapoints by subtracting the subject's actual motion thresholds from the motion thresholds that would be expected for a given age range (Kraft et al., 2010b). A negative result of this difference signifies that the motion sensitivity was below the age-norm, i.e., performing poorer than the age-norm. A positive difference of this age correction indicates a performance above the age-norm. This deviation from the age-norm of motion sensitivity will be referred to as " $\Delta$ motion sensitivity" 
and was entered as the depending variable into the statistical analyses.

\section{TRANSCRANIAL DIRECT CURRENT STIMULATION: ELECTRODE POSITION}

In order to control for individual variation in skull and posterior lobe anatomy, to maximize stimulation of the primary visual cortex and to provide a clinically relevant stimulation setting, the scalp directly superficial to the ipsilesional calcarine sulcus was selected to be the site of the stimulation electrode (Figure 2). Positioning was achieved using the navigation-system Nexstim Eximia Navigated Brain Stimulation System (Nexstim, Helsinki, Finland; see Schmidt et al., 2009), allowing co-registration of the subjects' heads in 3-D space with the corresponding anatomical MRI data using an infra-red camera (Polaris Spectra, Northern Digital Inc., ON, Canada) and $38 \mathrm{~mm}$ infra-red spectacles (Oculus, Wetzlar, Germany). A 1.5 T Magnetom Vision MRI scanner (Siemens, Erlangen, Germany) was used to acquire T1-weighted magnetizationprepared rapid gradient-echo sampling (MP RAGE) sequences for each subject (Brant-Zawadzki and Gillan, 1992; Howarth et al.,
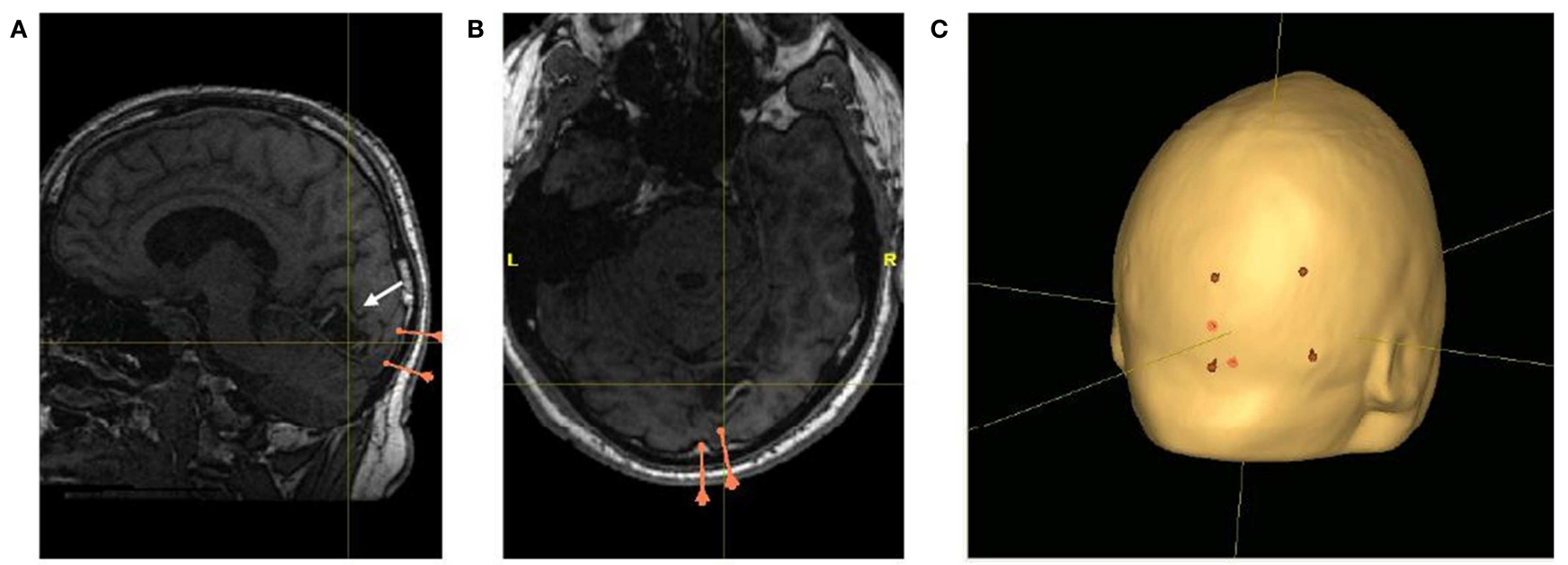

\section{Block 1}

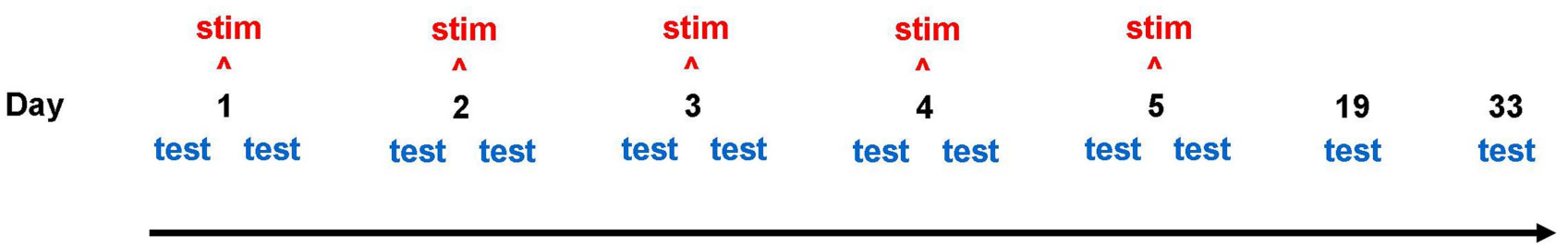

Inter-block interval: 16 days

\section{Block 2}

\begin{tabular}{|c|c|c|c|c|c|c|c|}
\hline & stim & stim & stim & stim & stim & & \\
\hline \multirow[t]{2}{*}{ Day } & 1 & 2 & 3 & 4 & 5 & 19 & 33 \\
\hline & test test & test test & test test & test test & test test & test & test \\
\hline
\end{tabular}

FIGURE 2 | Neuronavigation and study design. Screenshots from the neuronavigational software Nexstim Eximia. (A,B) Occipital lobe lesion in one subject (center of cross-hairs). Pink markers represent the two meningeal landmarks used for electrode positioning. (C)
Scalp localization (red) of the corners of the $5 \mathrm{~cm} \times 7 \mathrm{~cm}$ stimulation electrode above the ipsilesional calcarine sulcus (white arrow). Cross-hair location is constant in (A-C). The study design is illustrated in (D) 
2006). The lower horizontal border of the $5 \mathrm{~cm} \times 5 \mathrm{~cm}$ anodal electrode was defined by a scalp point superficial to the tentorium cerebelli; the medial vertical border of the anodal electrode position was defined by a scalp point superficial to the brain location $1 \mathrm{~cm}$ lateral to the interhemispheric falx cerebri (Figure 2). The midpoint of the $7 \mathrm{~cm} \times 5 \mathrm{~cm}$ reference cathodal electrode lay over the 20-10 electrode position $\mathrm{Cz}$. Electrode positions were delineated with permanent marker pen on the subject's head and renewed at the start of each intervention day to maintain correct electrode position without daily MRI-navigation.

\section{STIMULATION}

Anodal and sham tDCS was administered using an Eldith Direct Current Stimulator Plus (Neuroconn, Ilmenau, Germany), via two non-metallic conductive rubber electrodes ensheathed in synthetic sponges, which were soaked in $0.9 \% \mathrm{Na}^{+} \mathrm{Cl}^{-}$to reduce electrical resistance and subject discomfort, a key requirement of double-blind sham-controlled tDCS studies (Gandiga et al., 2006; Dundas et al., 2007). This solution was also carefully applied to subjects' scalps at electrode positions, and large hair strands dispersed. Stimulation was to cease automatically if circuit resistance exceeded $40 \mathrm{k} \Omega$; outwith the initial and final $15 \mathrm{~s}$ of stimulation (current "ramping" phase, see below) resistance did not exceed $8 \mathrm{k} \Omega$ for any subject. Electrodes were secured in place with a non-rubber headband and self-adhesive medical bandage. The stimulating electrode had a surface area of $25 \mathrm{~cm}^{2}$, the reference of $35 \mathrm{~cm}^{2}$.

Anodal and sham tDCS were administered for $20 \mathrm{~min}$ during stimulation sessions. Anodal tDCS was applied at a current of $1.5 \mathrm{~mA}$, giving a current density of $0.06 \mathrm{~mA} / \mathrm{cm}^{2}$, within published safety recommendations (Iyer et al., 2005; Poreisz et al., 2007; Williams et al., 2009; Kessler et al., 2012). At this current density, an initial skin tingling under the anodal electrode may be expected on initiation of tDCS (Paulus, 2003). For this reason, gradual current "ramping" was employed in the first $15 \mathrm{~s}$ of both anodal and sham tDCS (Hummel et al., 2008), after which $1.5 \mathrm{~mA}$ stimulation followed for $20 \mathrm{~min}$ in the anodal condition and no stimulation followed for $20 \mathrm{~min}$ in the sham condition. Current was ramped down for the final $15 \mathrm{~s}$ at the end of each 20-min sham and anodal stimulation session. Each 5-day intervention week was allocated a five-digit code, corresponding either to anodal or sham stimulation, by a supervising researcher who did not perform any experimental work. Thus the direct investigators were experimentally blinded to the stimulation condition. Subjects were informed of possible mild side-effects such as skin irritation, tingling under the electrodes, and mild headache, and encouraged to report any unpleasantness or discomfort.

\section{STUDY DESIGN}

The study followed a within-subject, repeated-measures, crossover design, comprising two blocks, each with a 5-day stimulation week with daily measurements, then two follow-up measurements at 2 and 4 weeks (Figure 2: Study Design). Subjects received either anodal or sham tDCS on five consecutive days, with visual testing before and after intervention. Measurements are described below with reference to study day and as "pre" or "post" (before or after intervention, only relevant for days 1-5). Subjects returned
2 weeks (day 19) and 4 weeks (day 33) later for follow-up measurements. This gave a total of 12 measurements per block (Figure 2). Long-term effects of anodal tDCS lasting up to 33 days were considered possible. To minimize the possibility of long-term effects of anodal tDCS (if administered in the first block) influencing the sham condition, a 16-day interval was observed between the last measurement of block one and the first stimulation day of block two; thus the total time interval between 5-day intervention weeks was 6 weeks, and total study duration per subject was 12 weeks (Figure 2). The second block followed an identical schedule as the first. Each subject received both anodal and sham intervention weeks and the two stimulation conditions were counterbalanced between blocks: six subjects received anodal tDCS in the first block, while six received it in the second block.

\section{DATA ANALYSIS}

All analyses were performed with IBM SPSS Statistics 19 for Windows. Age-corrected motion threshold values, generated by the subtraction of the subjects' motion threshold from decadesensitive normal reference values data $(=\Delta$ motion sensitivity, see above), were used for analysis. Due to a small number of missing values (see Results), using a traditional statistical analysis such as ANOVA with repeated measures would have led to a substantial decrease in statistical power. As an alternative statistical analysis of $\Delta$ motion sensitivity that is unaffected by few missing data, a linear mixed model (LMM) with subjects as random factor was used (Shek and Ma, 2011). The following variables were used to setup our statistical models. The covariate "stimulation" was coded 0 for sham and 1 for anodal stimulation. The covariate "time" was coded 1, 2, 3, 4, 5, 19, and 33 for the respective days. The covariate "sequence" was coded as 0 for the first block and 1 for the second block. The covariate "intervention" was coded as 0 for the measurement before the stimulation and 1 the measurement afterward. The covariate "quadrant" was coded as 0 for the lower quadrant and 1 for the upper quadrant. A $t$-test for independent groups was used to analyze potential baseline differences between the groups that started either with anodal tDCS or sham tDCS. In addition, a bivariate correlation analysis was performed to test whether the stimulation effect correlated with the lesion volume or the distance from the posterior lesion margin to the internal surface of the occipital pole cranium (Pearson correlation, two-tailed, $p<0.05)$. The stimulation effect was calculated across the stimulation week as the difference between baseline measurement (day 1 , before stimulation) and $\Delta$ motion sensitivity at the last day of the stimulation week (day 5, after stimulation). Accordingly, the long-term stimulation effect was calculated as the difference between baseline measurement (day 1 , before stimulation) and $\Delta$ motion sensitivity at the last day of the follow-up measurement (day 33).

\section{RESULTS}

Blinding to stimulation condition was successful: nine subjects reported not knowing whether they received anodal or sham tDCS in each block; of the remaining three, only one guessed correctly, with "moderate certainty," not because of feeling the stimulation, but due to a subjective improvement in peripheral visual field sensitivity within the anodal block. 
All procedures were well-tolerated by subjects and no serious known side-effects of tDCS were reported at any point. One subject with pre-existing scalp xerosis reported irritation during intervention sessions in both anodal and sham blocks, most likely due to $0.9 \% \mathrm{Na}^{+} \mathrm{Cl}^{-}$solution. A second subject with a previous history of intermittent piercing chest pain experienced a recurrence of chest pain on the third day of the sham block, not thought to be connected to the present study. No subjects reported deterioration in vision (sensitivity, acuity, resolution, color, movement, or general vision).

The parameter $\Delta$ motion sensitivity obtained at all measurements by all 12 subjects was approximately normally distributed. From the 576 possible total data points ( 12 subjects $\times 12$ time points $\times 2$ stimulations $\times 2$ unaffected quadrants), 10 measurements $(1.74 \%)$ were lost because a subject could not attend on the scheduled day. A $t$-test for independent groups did not reveal a significant difference in motor sensitivity between the anodal and sham condition at baseline of the first block $(p=0.266)$.

The LMM specified to model the effects of serial tDCS on $\Delta$ motion sensitivity within the stimulation week included the repeated covariates "stimulation" (anodal, sham), "intervention" (pre, post), "time" (day 1, day 2, day 3, day 4, day 5), and "quadrant" (superior, inferior) (Table 1). With regard to the within-subject design, the learning state between block one and block two differed (being less experienced in the first block and more experienced in the second block). Since this was expected to affect the performance of the motion detection task, the covariate "sequence" (block 1, block 2) was introduced into the LMM.

Significant main effects were found for "stimulation" $(p=0.05)$, and "time" $(p<0.01)$, i.e., $\Delta$ motion sensitivity increased across the stimulation week in both stimulation conditions (= learning effect), with an additional increase of $\Delta$ motion sensitivity for the anodal stimulation condition (Figure 3), even when a sequence effect is present with generally higher levels of $\Delta$ motion sensitivity in the second block $(p<0.01)$. Interestingly, the immediate intervention effect did not reach significance $(p=0.22)$, indicating that the improvement of $\Delta$ motion sensitivity was partially mediated through an overnight effect. There was a significant main effect for "quadrant" $(p<0.01)$, describing how greater changes in $\Delta$ motion sensitivity were attained in the upper visual field quadrant.

Table 1 | Linear mixed model statistics from the stimulation week of the study.

\begin{tabular}{lccc}
\hline Parameter & Estimate & Std. error & $\boldsymbol{p}$-Value \\
& & & \\
\hline Intercept & 0.90 & 2.62 & 0.74 \\
Stimulation & 1.11 & 0.57 & 0.05 \\
Time & 0.99 & 0.20 & $<0.01$ \\
Intervention & -0.71 & 0.57 & 0.22 \\
Quadrant & 2.76 & 0.57 & $<0.01$ \\
Sequence & 5.31 & 0.57 & $<0.01$ \\
Variance components & & SD & \\
Subjects & & 6.20 &
\end{tabular}

In order to analyze the potential long-term effects of serial tDCS, the parameter $\Delta$ motion sensitivity measured poststimulation was entered in another LMM (Table 2), including the covariates "time" (day 1 , day 2, day 3, day 4, day 5, day 19, day 33), "stimulation" (anodal, sham), and "quadrant" (superior, inferior). Only one test per day of stimulation block (poststimulation) was included, because the follow-up period comprises only post-stimulation measurements. In order to adjust for a sequence effect between blocks, the covariate "sequence" was included. Since the learning curve may decline throughout the experiment the interaction "sequence $\times$ time" was introduced into the LMM.

The covariate "stimulation" was significant $(p=0.05)$, as were the covariates "time", "sequence", and "quadrant" (all $p<0.01$ ); importantly, this confirms a significant long-term effect of serial anodal tDCS, again with higher levels of $\Delta$ motion sensitivity measured in the upper visual field (Figure 4).

The two-way interaction "sequence $\times$ time" yielded a significant interaction $(p<0.01)$, with a negative $\beta$-estimate indicating a ceiling effect of learning, declining within the follow-up period after the second block, which was not seen after the first block (Figure 4).

Improved performance did not decline between studies blocks, i.e., subjects started on a higher performance at the beginning of the second stimulation week. Subjects receiving sham tDCS in the

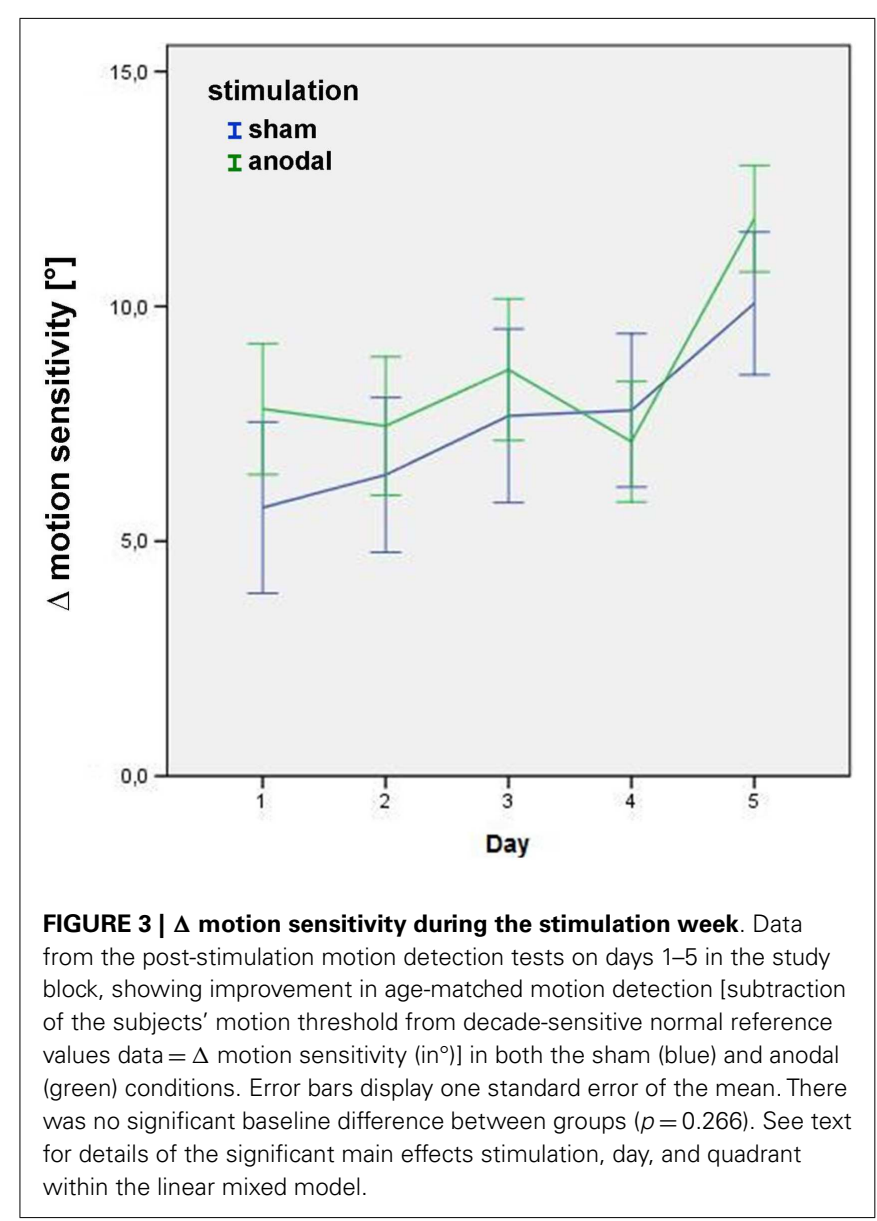


Table 2 | Linear mixed model statistics from stimulation week and follow-up measurements.

\begin{tabular}{lccr}
\hline Parameter & Estimate & Std. error & $\boldsymbol{p}$-Value \\
\hline Intercept & -3.96 & 2.90 & \\
Stimulation & 1.46 & 0.74 & 0.18 \\
Time & 0.39 & 0.10 & 0.05 \\
Quadrant & 3.27 & 0.74 & $<0.01$ \\
Sequence & 5.99 & 0.98 & $<0.01$ \\
Time $\times$ sequence & -0.18 & 0.07 & $<0.01$ \\
Variance components & & SD & \\
Subjects & & 6.72 &
\end{tabular}

second stimulation block, i.e., after the anodal tDCS block, could improve their performance across the second stimulation week to a lesser degree. This might be explained by a ceiling effect of the performance of the motion detection task.

None of the correlation analyses yielded a significant correlation between the stimulation effect (over the stimulation week or the follow-up period) and lesion specific parameters, i.e., the lesion volume or the minimum distance from the internal surface of the occipital pole cranium, and the stimulation effect $(p>0.05)$.

\section{DISCUSSION \\ SUMMARY OF RESULTS}

Serial anodal tDCS over the visual cortex has an additive effect on the performance of a visual perceptual detection task, measuring $\Delta$ motion sensitivity in the unaffected hemisphere of subjects with chronic stroke who received tDCS over their lesioned visual cortex. This effect was additional to a fast perceptual learning effect that was particularly present in the early phase of the experiment, but importantly was still measurable in the follow-up measurements 2 and 4 weeks after the last stimulation. $\Delta$ motion sensitivity could, however, still be increased by serial anodal tDCS in the later phase of the experiment in the group that received sham tDCS in the first block and in which the learning curve had already reached a plateau. No immediate effects of anodal tDCS on motion sensitivity were seen, and performance improvement was mediated through an overnight effect during the stimulation week.

\section{ALTERNATIVE ACCOUNTS}

Alternative explanations for the lack of an immediate stimulation effect might be that $\Delta$ motion sensitivity was measured too late. Depending on randomization, $\Delta$ motion sensitivity was measured 7-11 min after the end of the application of tDCS. However, this short delay can be considered unlikely to conceal a tDCS effect due to the findings of Antal et al. (2004b), who reported that with a less intense stimulation procedure of $1 \mathrm{~mA}$ for $10 \mathrm{~min}$ over V1, anodal tDCS led to a reduced moving TMS-phosphene threshold, a measure of excitability of MT, which was still significant $10 \mathrm{~min}$ after the anodal tDCS. Pre-existing differences in the subject groups caused by unsatisfactory randomization (e.g., variation in lesion characteristics) are unlikely, since baseline differences were non-significant and were additionally controlled for by the within-subject design. Successful double-blinding was achieved. The effect of serial sham tDCS, however, could not be measured directly since a learning effect was also present in the group that received sham stimulation in the first study block. The improvement of $\Delta$ motion sensitivity is most likely mediated by a direct stimulation of the contralesional V1 area through direct anatomical connections to V5/MT. In a previous study of our group (Kraft et al., 2010a), the effect of anodal and cathodal tDCS on contrast sensitivity, a V1 task, was unaffected by the precise site of the stimulating electrode, i.e., over the left or the right visual cortex (O1 or O2). This is most probably due to their close vicinity and the coarse focality of standard tDCS. The greater changes in $\Delta$ motion sensitivity measured in the upper visual field quadrant (represented below the calcarine sulcus) compared to the lower visual field quadrant (represented above the calcarine sulcus) also support this hypothesis. Given the electrode montage used, a higher current density could be expected in the infracalcarine V1, whereas the angle between the stimulating and reference electrodes is more tangential compared to the supracalcarine V1. Lesion specific parameters, such as the lesion volume and the minimum distance from the internal surface of the occipital pole cranium did not correlate with the stimulation effect of the contralesional unaffected hemifield.

\section{LONG-TERM EFFECTS OF SERIAL tDCS INTERACT WITH PERCEPTUAL LEARNING PROCESSES}

These data represent the first report of long-term effects of anodal tDCS on motion sensitivity, albeit without immediate behavioral effects. A possible explanation for this discrepancy may be that serial anodal tDCS interacts with parallel ongoing learning processes, fostering the stimulation effect over time, with both processes putatively mediated by NMDA-receptor-mediated plasticity (Stagg and Nitsche, 2011). The important interaction of learning and tDCS is highlighted in the small number of studies into serial tDCS that have shown encouraging results in the motor system. Serial anodal tDCS administered over M1 in healthy subjects on five consecutive days during motor task practice sessions significantly enhanced the learning of the complex motor skill task versus sham (Reis et al., 2009). In accordance with our pattern of results, gains were not immediate, but seen selectively between anodal stimulations (i.e., as overnight effects), and persisted throughout a 3-month follow-up phase. It may indeed be the complexity of the sequential visual isometric pinch paradigm used by Reis and colleagues, which required concerted activity of several primary and secondary motor regions, that allowed an elevation of cortical excitability to be translated into lasting behavioral effects, when simpler outcome measures such as specific muscle-group MEP thresholds were not potentiated by anodal tDCS repeated at a 24-h interval (Monte-Silva et al., 2013). More specifically, two 13min sessions of anodal tDCS administered over the motor-cortex with a 3 or 20 min gap between them lowered MEP thresholds, persistent at $24 \mathrm{~h}$; however, if the gap between the two anodal tDCS sessions was longer, at 3 or $24 \mathrm{~h}$, no persisting raised excitability was seen (Monte-Silva et al., 2013). These findings suggest that the combination of tDCS-modulated excitability with learning is crucial and task-dependent, and emphasize the need to look beyond immediate effects in understanding the potential of tDCS 
A

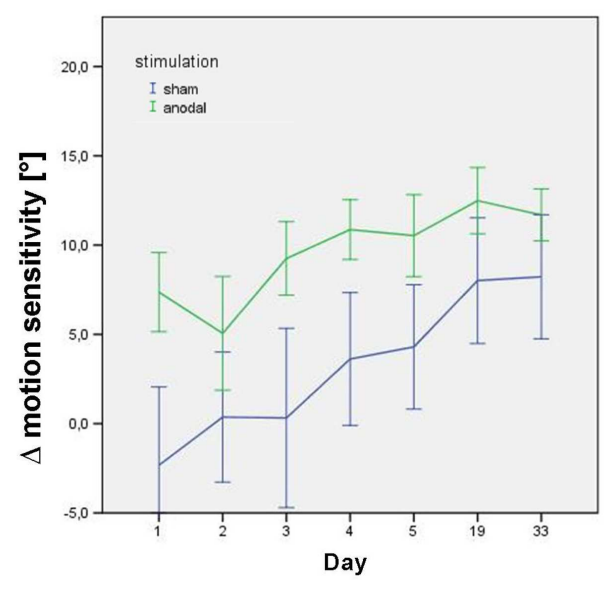

C

Block 2

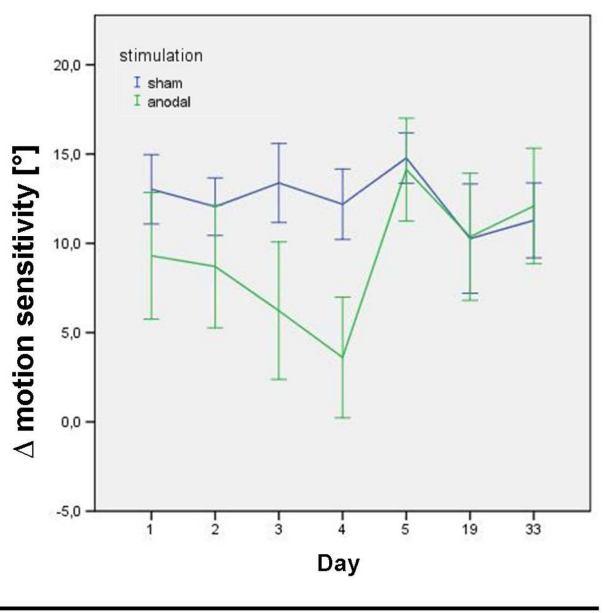

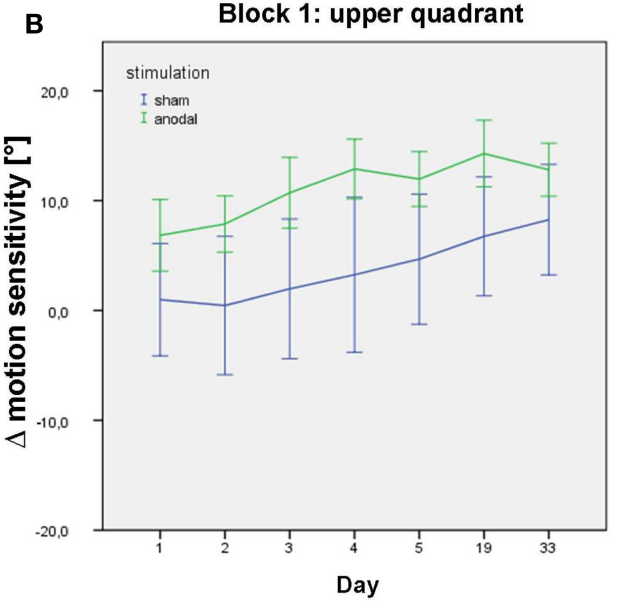

FIGURE 4 | $\Delta$ motion sensitivity during the stimulation week and follow-up. (A) Data from the post-stimulation motion detection tests on days 1-33 in the first study block, showing improvement in age-matched motion detection [subtraction of the subjects' motion threshold from decade-sensitive normal reference values data $=\Delta$ motion sensitivity $\left(\right.$ in $\left.\left.^{\circ}\right)\right]$ in both the sham (blue) and anodal (green) conditions during the stimulation week and follow-up. (B) Data from the post-stimulation motion detection tests on days 1-33 in the first study block for the upper and

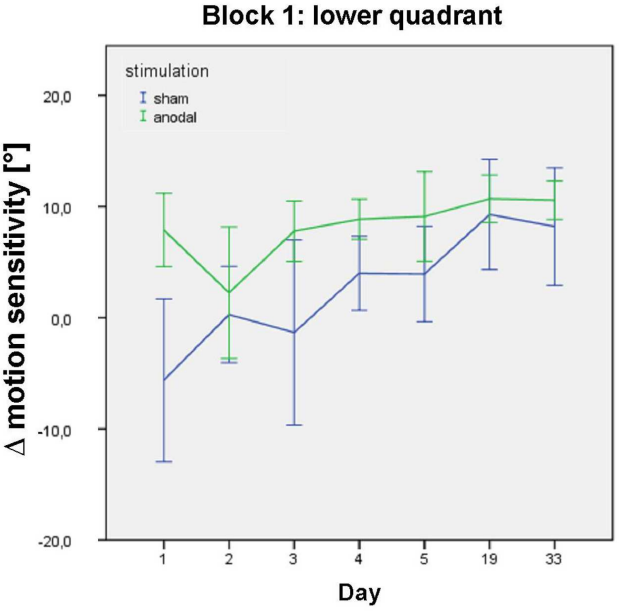

lower quadrant separately. (C) Data from the post-stimulation motion detection tests on days 1-33 in the second study block, showing maintenance of motion detection level in the sham condition (blue) over the intervention week (following anodal stimulation in block 1) and improvement in motion detection in the anodal condition (green) over the intervention week (following sham stimulation in block 1). Follow-up sessions show a similar decrease in motion detection in both groups. Error bars display one standard error of the mean. in long-term neuromodulation (Reis et al., 2009; Reis and Fritsch, 2011; Stagg and Nitsche, 2011).

\section{TEMPORAL DYNAMICS OF tDCS EFFECTS}

It is still a matter of debate whether there is a susceptible time window in which anodal tDCS has its optimal effect. Antal et al. (2004c); Antal and Paulus (2008) reported that the effects of tDCS on V5 are learning-phase dependent: cathodal and anodal tDCS both improved the concomitant learning of a complex visuomotor task in the early phase of learning only, but not later, emphasizing the importance of learning to the effects of tDCS on motion perception.
In contrast, the visuomotor learning paradigm of Reis et al. (2009) revealed a cumulative overnight benefit of anodal tDCS over repeated sessions of anodal stimulation. A possible explanation may be differing difficulties between the tasks applied in both studies.

In the present study, where visuomotor learning processes were of minor importance, perceptual learning processes were assumed to be inherently involved. The learning effect was not detectable immediately after stimulation, but evolved overnight. Serial anodal tDCS over V1 interacted with the process of perceptual learning when administered both in an early learning state (first stimulation week) and in a later over-learned state 
(second stimulation week). In a psychophysical study of the learning dynamics of the visual submodalities used here, the same motion detection task was practised over 5 days in healthy young subjects (Kraft et al., 2010b). Increasing test difficulty by changing the task to a motion discrimination task effectively flattened the steepness of the learning curve. These findings therefore suggest that task demands influence the time dynamics of perceptual learning processes: it is likely that the difficulty of a task in a repeated tDCS paradigm may contribute substantially to the dynamics of the tDCS effects. Improvements in functional output are likely to come through the integration of tDCS-induced plasticity with task learning, training, and other endogenic plasticity mechanisms.

The present study contrasts on several grounds with the four published studies investigating repeated tDCS in the visual system, all describing one small clinical trial $(n=4$ per treatment condition) into combined anodal tDCS and visual restoration therapy (VRT), which utilized sparser measurement timepoints at baseline and monthly follow-ups (Halko et al., 2011; Plow et al., 2011, 2012a,b). Although significant improvements compared to sham tDCS with VRT were reported, and fMRI correlates of neuroplastic change were demonstrated in a single case study (Halko et al., 2011), potentially representing encouraging

\section{REFERENCES}

Accornero, N., Li Voti, P., La Riccia, M., and Gregori, B. (2007). Visual evoked potentials modulation during direct current cortical polarization. Exp. Brain Res. 178, 261-266. doi:10.1007/s00221-006-0733-y

Antal, A., Kincses, T. Z., Nitsche, M. A., Bartfai, O., and Paulus, W. (2004a). Excitability changes induced in the human primary visual cortex by transcranial direct current stimulation: direct electrophysiological evidence. Invest. Ophthalmol. Vis. Sci. 45, 702-707. doi:10.1167/iovs.030688

Antal, A., Varga, E. T., Nitsche, M. A., Chadaide, Z., Paulus, W., Kovács, G., et al. (2004b). Direct current stimulation over MT+/V5 modulates motion aftereffect in humans. Neuroreport 15, 2491-2494. doi:10.1097/00001756-20041115000012

Antal, A., Nitsche, M. A., Kruse, W., Kincses, T. Z., Hoffmann, K. P., and Paulus, W. (2004c). Direct current stimulation over V5 enhances visuomotor coordination by improving motion perception in humans. J. Cogn. Neurosci. 16, 521-527. doi:10.1162/089892904323057263

Antal, A., Begemeier, S., Nitsche, M. A., and Paulus, W. (2008). Prior state of cortical activity influences subsequent practicing of a visuomotor coordination task. Neuropsychologia 46, 3157-3161. doi:10.1016/j.neuropsychologia. 2008.07.007
Antal, A., Nitsche, M. A., and Paulus, W. (2001). External modulation of visual perception in humans. Neuroreport 12, 3553-3555. doi:10.1097/00001756-20011116000036

Antal, A., and Paulus, W. (2008). Transcranial direct current stimulation and visual perception. Perception 37, 367-374. doi:10.1068/p5872

Antal, A., Paulus, W., and Nitsche, M. A. (2011). Electrical stimulation and visual network plasticity. Restor. Neurol. Neurosci. 29, 365-374. doi:10.3233/RNN-2011-0609

Bailey, C. H., Giustetto, M., Huang, Y. Y., Hawkins, R. D., and Kandel, E. R. (2000). Is heterosynaptic modulation essential for stabilizing Hebbian plasticity and memory? Nat. Rev. Neurosci. 1, 11-20. doi:10.1038/35036191

Bindman, L. J., Lippold, O. C., and Redfearn, J. W. (1962). Long-lasting changes in the level of the electrical activity of the cerebral cortex produced by polarizing currents. Nature 196, 584-585. doi:10.1038/196584a0

Bindman, L. J., Lippold, O. C., and Redfearn, J. W. (1964). The action of polarizing currents on the cerebral cortex of the rat (1) during current flow and (2) in the production of long-lasting after-effects. J. Physiol. (Lond.) 172, 369-382.

Bonnefond, A., Rohmer, O., Hoeft, A., Muzet, A., and Tassi, P. (2003). Interaction of age with time of day and mental load in different cognitive tasks. Percept. Mot.

indications of neuroplasticity in the lesioned visual cortex, the studies do not greatly inform our understanding of the shortand medium-term timecourse, or nature of isolated tDCS-specific effects in the visual system.

\section{CONCLUSION}

The temporal dynamics of long-term (days to weeks) tDCS effects are of general importance to the field of tDCS. Here, we provided evidence for long-term changes of $\Delta$ motion sensitivity within the stroke-unaffected hemifield induced by serial anodal tDCS that continue to increase during a 4-week follow-up period. Improving the visual perceptual function of residual intact neurons in the visual system may contribute as a compensational strategy for deficient visual function of stroke-related neuronal loss. Future research efforts should examine the clinical relevance of these findings and focus on the closer temporal and spatial dynamics of serial tDCS with a progression to investigating and further understanding the physiology of clinically applied tDCS within functionally impaired neural systems.

\section{ACKNOWLEDGMENTS}

We wish to thank the Else Kröner-Fresenius-Stiftung for funding this study.

Skills 96(3 Pt 2), 1223-1236. doi:10.2466/PMS.96.4.1223-1236

Brant-Zawadzki, M., and Gillan, G. D. (1992). MP RAGE a three-dimensional, T1-weighted, gradient-echo sequence. Radiology 182, 769-775.

Creutzfeldt, O. D., Fromm, G. H., and Kapp, H. (1962). Influence of transcortical d-c currents on cortical neuronal activity. Exp. Neurol. 5, 436-452. doi:10.1016/00144886(62)90056-0

DeCarli, C., Kawas, C., Morrison, J. H., Reuter-Lorenz, P. A., Sperling, R. A., and Wright, C. B. (2012). Session II: mechanisms of age-related cognitive change and targets for intervention: neural circuits, networks, and plasticity. J. Gerontol. A Biol. Sci. Med. Sci. 67, 747-753. doi:10.1093/gerona/gls111

Dundas, J. E., Thickbroom, G. W. and Mastaglia, F. L. (2007). Perception of comfort during transcranial DC stimulation: effect of $\mathrm{NaCl}$ solution concentration applied to sponge electrodes. Clin. Neurophysiol. 118, 1166-1170. doi:10.1016/j.clinph.2007.01.010

Furubayashi, T., Terao, Y., Arai, N., Okabe, S., Mochizuki, H., Hanajima, R., et al. (2008). Short and long duration transcranial direct current stimulation (tDCS) over the human hand motor area. Exp. Brain Res. 185, 279-286. doi:10.1007/s00221007-1149-z

Gandiga, P. C., Hummel, F. C., and Cohen, L. G. (2006). Transcranial
DC stimulation (tDCS): a tool for double-blind sham-controlled clinical studies in brain stimulation. Clin. Neurophysiol. 117, 845-850. doi:10.1016/j.clinph.2005.12.003

Gartside, I. B. (1968). Mechanisms of sustained increases of firing rate of neurones in the rat cerebral cortex after polarization: role of protein synthesis. Nature 220, 383-384. doi:10.1038/220383a0

Grady, C. (2012). The cognitive neuroscience of ageing. Nat. Rev. Neurosci. 13, 491-505. doi:10.1038/nrn3256

Halko, M. A., Datta, A., Plow, E. B., Scaturro, J., Bikson, M., and Merabet, L. B. (2011). Neuroplastic changes following rehabilitative training correlate with regional electrical field induced with tDCS. Neuroimage 57, 885-891. doi:10.1016/j.neuroimage.2011. 05.026

Howarth, C., Hutton, C., and Deichmann, R. (2006). Improvement of the quality of T1-weighted anatomical brain scans. $\mathrm{Neu}$ roimage 29, 930-937. doi:10. 1016/j.neuroimage.2005.08.004

Hummel, F. C., Celnik, P., PascualLeone, A., Fregni, F., Byblow, W. D., Buetefisch, C. M., et al. (2008). Controversy: noninvasive and invasive cortical stimulation show efficacy in treating stroke patients. Brain Stimul. 1, 370-382. doi:10.1016/j.brs.2008.09.003

Iyer, M. B., Mattu, U., Grafman, J., Lomarev, M., Sato, S., and Wassermann, E. M. (2005). Safety 
and cognitive effect of frontal DC brain polarization in healthy individuals. Neurology 64, 872-875. doi:10.1212/01.WNL.0000152986. 07469.E9

Kessler, S. K., Turkeltaub, P. E., Benson, J. G., and Hamilton, R. H. (2012). Differences in the experience of active and sham transcranial direct current stimulation. Brain Stimul. 5, 155-162. doi:10.1016/j.brs.2011.02.007

Kraft, A., Roehmel, J., Olma, M. C., Schmidt, S., Irlbacher, K., and Brandt, S. A. (2010a). Transcranial direct current stimulation affects visual perception measured by threshold perimetry. Exp. Brain Res. 207, 283-290. doi:10.1007/s00221010-2453-6

Kraft, A., Grimsen, C., Trenner, D., Kehrer, S., Lipfert, A., Köhnlein, M., et al. (2010b). Specificity of fast perceptual learning in shape localisation tasks based on detection versus form discrimination. Vision Res. 50, 473-478. doi:10.1016/j.visres.2009.12.011

Landau, W. M., Bishop, G. H., and Clare, M. H. (1964). Analysis of the form and distribution of evoked cortical potentials under the influence of polarizing currents. J. Neurophysiol. 27, 788-813.

Lang, N., Siebner, H. R., Chadaide, Z., Boros, K., Nitsche, M. A., Rothwell, J. C., et al. (2007). Bidirectional modulation of primary visual cortex excitability: a combined tDCS and rTMS study. Invest. Ophthalmol. Vis. Sci. 48, 5782-5787. doi:10.1167/iovs.07-0706

Liebetanz, D., Nitsche, M. A., Tergau, F., and Paulus, W. (2002). Pharmacological approach to the mechanisms of transcranial DCstimulation-induced after-effects of human motor cortex excitability. Brain 125(Pt 10), 2238-2247. doi:10.1093/brain/awf238

Monte-Silva, K., Kuo, M. F., Hessenthaler, S., Fresnoza, S., Liebe$\operatorname{tanz}$, D., Paulus, W., et al. (2013). Induction of late LTP-like plasticity in the human motor cortex by repeated non-invasive brain stimulation. Brain Stimul. 6, 424-432. doi:10.1016/j.brs.2012.04.011

Nasreddine, Z. S., Phillips, N. A., Bédirian, V., Charbonneau, S., Whitehead, V., Collin, I., et al. (2005). The Montreal Cognitive Assessment, MoCA: a brief screening tool for mild cognitive impairment. J. Am. Geriatr. Soc. 53, 695-699. doi:10.1111/j.15325415.2005.53221.x

Nitsche, M. A., Cohen, L. G., Wassermann, E. M., Priori, A., Lang, N., Antal, A., et al. (2008). Transcranial direct current stimulation: state of the art 2008. Brain Stimul. 1, 206-223. doi:10.1016/j.brs.2008.06.004

Nitsche, M. A., Fricke, K., Henschke, U., Schlitterlau, A., Liebetanz, D., Lang, N., et al. (2003a). Pharmacological modulation of cortical excitability shifts induced by transcranial direct current stimulation in humans. J. Physiol. (Lond.) 553(Pt 1), 293-301. doi:10.1113/ jphysiol.2003.049916

Nitsche, M. A., Grundey, J., Liebetanz, D., Lang, N., Tergau, F., and Paulus, W. (2004). Catecholaminergic consolidation of motor cortical neuroplasticity in humans. Cereb. Cortex 14, 1240-1245. doi:10.1093/cercor/bhh085

Nitsche, M. A., and Paulus, W. (2000). Excitability changes induced in the human motor cortex by weak transcranial direct current stimulation. J. Physiol. (Lond.) 527(Pt 3), 633-639. doi:10.1111/j.14697793.2000.t01-1-00633.x

Nitsche, M. A., and Paulus, W. (2001). Sustained excitability elevations induced by transcranial DC motor cortex stimulation in humans. Neurology 57, 1899-1901. doi:10.1212/WNL.57.10.1899

Olma, M. C., Kraft, A., Roehmel, J., Irlbacher, K., and Brandt, S. A. (2011). Excitability changes in the visual cortex quantified with signal detection analysis. Restor. Neurol. Neurosci. 29, 453-461. doi:10.3233/RNN-2011-0607

Paulus, W. (2003). Transcranial direct current stimulation (tDCS). Suppl. Clin. Neurophysiol. 56, 249-254. doi:10.1016/S1567-424X(09)70 229-6

Plow, E. B., Obretenova, S. N., Halko, M. A., Kenkel, S., Jackson, M. L., Pascual-Leone, A., et al. (2011). Combining visual rehabilitative training and noninvasive brain stimulation to enhance visual function in patients with hemianopia: a comparative case study. $P M \quad R \quad 3,825-835$. doi:10.1016/j.pmrj.2011.05.026

Plow, E. B., Obretenova, S. N., Jackson, M. L., and Merabet, L. B. (2012a). Temporal profile of functional visual rehabilitative outcomes modulated by transcranial direct current stimulation. Neuromodulation 15, 367-373. doi:10.1111/j.15251403.2012.00440.x

Plow, E. B., Obretenova, S. N., Fregni, F., Pascual-Leone, A., and Merabet, L. B. (2012b). Comparison of visual field training for hemianopia with active versus sham transcranial direct cortical stimulation. Neurorehabil. Neural. Repair 26, 616-626. doi:10.1177/1545968311431963

Poreisz, C., Boros, K., Antal, A., and Paulus, W. (2007). Safety aspects of transcranial direct current stimulation concerning healthy subjects and patients. Brain Res. Bull. 72, 208-214. doi:10. 1016/j.brainresbull.2007.01.004

Priori, A., Berardelli, A., Rona, S., and Manfredi, M. (1998). Polarization of the human motor cortex through the scalp. Neuroreport 9 2257-2260. doi:10.1097/00001756199807130-00020

Quartarone, A., Morgante, F., Bagnato, S., Rizzo, V., Sant'Angelo, A., Aiello, E., et al. (2004). Long lasting effects of transcranial direct current stimulation on motor imagery. Neuroreport 15, 1287-1291. doi:10. 1097/01.wnr.0000127637.22805.7c

Reis, J., and Fritsch, B. (2011). Modulation of motor performance and motor learning by transcranial direct current stimulation. Curr. Opin. Neurol. 24, 590-596. doi:10. 1097/WCO.0b013e32834c3db0

Reis, J., Schambra, H. M., Cohen, L. G., Buch, E. R., Fritsch, B., Zarahn, E., et al. (2009). Noninvasive cortical stimulation enhances motor skill acquisition over multiple days through an effect on consolidation. Proc. Natl. Acad. Sci. U.S.A. 106, 1590-1595. doi:10.1073/pnas.0805413106

Rosenkranz, K., Nitsche, M. A., Tergau, F., and Paulus, W. (2000). Diminution of training-induced transient motor cortex plasticity by weak transcranial direct current stimulation in the human. Neurosci. Lett. 296, 61-63. doi:10.1016/S03043940(00)01621-9

Schmidt, C., Collette, F., Cajochen, C., and Peigneux, P. A. (2007). Time to think: circadian rhythms in human cognition. Cogn. Neuropsychol. 24, 755-789. doi:10.1080/02643290701754158

Schmidt, S., Cichy, R. M., Kraft, A., Brocke, J., Irlbacher, K., and
Brandt, S. A. (2009). An initial transient-state and reliable measures of corticospinal excitability in TMS studies. Clin. Neurophysiol. 120, 987-993. doi:10.1016/j.clinph.2009. 02.164

Shek, D. T., and Ma, C. M. (2011). Longitudinal data analyses using linear mixed models in SPSS: concepts, procedures and illustrations. ScientificWorldJournal 11, 42-76. doi:10.1100/tsw.2011.2

Stagg, C. J., and Nitsche, M. A. (2011). Physiological basis of transcranial direct current stimulation. Neuroscientist 17, 37-53. doi:10.1177/1073858410386614

Watson, A. B., and Pelli, D. G. (1983). QUEST: a Bayesian adaptive psychometric method. Percept. Psychophys. 33, 113-120. doi:10.3758/BF03202828

Williams, J. A., Imamura, M., and Fregni, F. (2009). Updates on the use of non-invasive brain stimulation in physical and rehabilitation medicine. J. Rehabil. Med. 41, 305-311. doi:10.2340/16501977-0356

Zhang, X., Kedar, S., Lynn, M. J., Newman, N. J., and Biousse, V. (2006). Natural history of homonymous hemianopia. Neurology 66, 901-905. doi:10.1212/01.wnl.0000203338.54 323.22

Conflict of Interest Statement: The authors declare that the research was conducted in the absence of any commercial or financial relationships that could be construed as a potential conflict of interest.

Received: 25 February 2013; accepted: 10 June 2013; published online: 24 June 2013.

Citation: Olma MC, Dargie RA, Behrens $J R$, Kraft A, Irlbacher K, Fahle $M$ and Brandt SA (2013) Long-term effects of serial anodal tDCS on motion perception in subjects with occipital stroke measured in the unaffected visual hemifield. Front. Hum. Neurosci. 7:314. doi: 10.3389/fnhum.2013.00314

Copyright (C) 2013 Olma, Dargie, Behrens, Kraft, Irlbacher, Fahle and Brandt. This is an open-access article distributed under the terms of the Creative Commons Attribution License, which permits use, distribution and reproduction in other forums, provided the original authors and source are credited and subject to any copyright notices concerning any third-party graphics etc. 